\title{
Cytogenetic abnormality in patients with multiple myeloma analyzed by fluorescent in situ hybridization
}

This article was published in the following Dove Press journal:

OncoTargets and Therapy

3 March 2016

Number of times this article has been viewed

Ying $\mathrm{Hu}$

Wenming Chen

Shilun Chen

Zhongxia Huang

Department of Hematology, Beijing Chaoyang Hospital Affiliated to

Capital Medical University, Beijing,

People's Republic of China
Correspondence: Wenming Chen Department of Hematology, Beijing Chaoyang Hospital Affiliated to Capital Medical University, No 5 Jingyuan Road, Beijing 100043, People's Republic of China

Email13910107759@I63.com
Objective: To analyze the fluorescent in situ hybridization (FISH) data and the association with clinical characteristics, therapy response, and survival time in patients with multiple myeloma. Method: We performed a retrospective review of patients with multiple myeloma from November 2010 to April 2014.

Results: Cytogenetic abnormalities by FISH were detectable in $66 \%$ of patients. One cytogenetic abnormality, two cytogenetic abnormalities, and complex abnormalities were detectable in $21.2 \%$, $51.5 \%$, and $27.3 \%$ of cases, respectively. $1 \mathrm{q} 21$ amplification, $\mathrm{t}(4 \mathrm{p} 16.3 / 14 \mathrm{q} 32)$, and $17 \mathrm{p}$ deletion were observed in $69.7 \%, 30.3 \%$, and $21.2 \%$ of cases, respectively. Total response rates (complete response $[\mathrm{CR}]+$ near CR + partial response) were $93.8 \%$ and $82.1 \%$, respectively, in cytogenetic normality group and abnormality group. CR rates were $50 \%$ and $32.1 \%$, respectively. Median overall survival (OS) time was 51 months and 24 months, respectively, in cytogenetic normality group and abnormality group $(P<0.05)$. Median OS time was not significantly different between 1q21 amplification group and no 1q21 amplification group in patients with FISH abnormalities $(P>0.05)$. Median OS time was not significantly different between $\mathrm{t}(4 ; 14)$ group and no $\mathrm{t}(4 ; 14)$ group in patients with FISH abnormalities $(P>0.05)$. Seven patients of $17 \mathrm{p}$ deletion died in 2 years.

Conclusion: Multiple myeloma is characterized by a high occurrence of chromosomal aberrations. 1q21 amplification and $\mathrm{t}(4 ; 14)$ are the most common abnormalities. Multiple cytogenetic abnormalities are frequently observed in the same one patient. The total response rate, CR rate, and OS time are worse in cytogenetic abnormal patients compared with cytogenetic normal patients. Patients with $17 \mathrm{p}$ deletion have a very poor prognosis. Future goals of therapy will be to achieve minimal residual disease, biomarkers, and genomic data, which might provide a better estimate of the depth of response to therapy and OS.

Keywords: multiple myeloma, FISH, clinical characteristics, survival time

\section{Introduction}

Cytogenetic abnormalities have important prognostic values for multiple myeloma, especially in distinguishing the high-risk patients. Cytogenetic abnormalities were detected by fluorescent in situ hybridization (FISH) in most patients with newly diagnosed multiple myeloma (MM). MM is a heterogeneous disease with different cytogenetic aberrations, clinical characteristics, and therapy responses. Cytogenetic abnormalities are generally associated with prognosis.

\section{Method}

Samples for this study were acquired with written informed patient consent, in compliance with Beijing Chaoyang Hospital Institutional Review Board. We identified 
50 patients (22 males, 28 females) with newly diagnosed MM based on the FISH data between November 2010 and April 2014. Median age of them was 62 years (range: 38-79 years). Diagnosis criteria were as defined by the World Health Organization (2001) diagnostic criteria. The examinations included immunoglobulin quantity, immunofixation electrophoresis, bone marrow hemocytology, bone marrow immunophenotype, FISH, X-ray/computed tomography/ magnetic resonance imaging, complete blood cell count, Scr, $\beta 2$-microglobulin (MG), lactate dehydrogenase (LDH), and C-reactive protein (CRP). All the patients were treated with bortezomib-based chemotherapy regimens. Eight patients were treated with lenalidomide. Response criteria were as defined by the International Myeloma Working Group uniform response criteria. The two groups of patients (cytogenetically abnormal vs cytogenetically normal) were evaluated for median overall survival (OS).

\section{Results}

FISH probes used in the study included FGFR3/IGH, CCND1/IGH, IGH/MAF, 1q21, and TP53. Cytogenetic aberrations were found in 66\% (33 of 50) of patients. According to the Durie-Salmon staging system: one was in stage I, 20 were in stage IIIA, and 12 were in stage IIIB, and as per International Staging System (ISS): three were in stage I, seven were in stage II, and 23 were in stage III. Twelve were with $\lg$ G type, ten were with $\lg$ A type, and eleven were with light-chain type. Also, twenty-three cases were analyzed by flow cytometry. Positive expressions of CD38, CD138, CD56, CD45, CD2, CD20, CD28, CD22, and CD117 were detected in $100 \%, 100 \%, 73.9 \%, 34.8 \%, 34.8 \%, 30.4 \%$, $21.7 \%, 17.4 \%$, and $17.4 \%$ of cases, respectively. Multiple bone lesions were seen in $97 \%$ of patients. $\beta 2-\mathrm{MG}$ exceeding $3.5 \mathrm{mg} / \mathrm{dL}$ was seen in $73.3 \%$ of patients. Hypoproteinemia was seen in $56.7 \%$ of patients. Anemia was seen in $56.7 \%$ of patients. Thrombopenia was seen in $26.7 \%$ of patients. Elevated serum creatinine was seen in $30 \%$ of patients. Elevated CRP was seen in $38.5 \%$ of patients. Elevated LDH was seen in $20.8 \%$ of patients. Hypercalcemia was seen in $26.7 \%$ of patients. Elevated erythrocyte sedimentation rate was seen in $70.8 \%$ of patients (Table 1 ). One cytogenetic aberration was detected in $21.2 \%$ of patients. Two cytogenetic aberrations were detected in $51.5 \%$ of patients. Multiple cytogenetic aberrations were detected in $27.3 \%$ of patients. 1q21 amplification was seen in 69.7\% (23 of 33) of patients. $t(4 p 16.3 / 14 q 32)$ was seen in $30.3 \%$ (ten of 33 ) of patients. $14 \mathrm{q} 32$ amplification/deletion was detected in $27.3 \%$ (nine of 33 ) of patients. 17p deletion was seen in $21.2 \%$ (seven of 33) of patients. 16q23 deletion/amplification was seen in $21.2 \%$ (seven of 33 ) of patients. $t(14 q 32 / 11 q 13)$ was detected in $15.5 \%$ (five of 33 ) of patients. 11q13 amplification was detected in $18.2 \%$ (six of 33 ) of patients. 4 p16.3 deletion was detected in $3 \%$ (one of 33 ) of patients. A dual fusion probe for IGH and a dual fusion probe for CCND1 were used in our study. A translocation with an unknown partner will give an extra signal. So an extra signal is not necessarily indicative of amplification.

\section{Response and prognosis}

Novel agents including thalidomide, lenalidomide, and bortezomib were used for induction or salvage treatment. Patients were also treated with vindesine, adriamycin, dexamethasone (VAD), vindesine, liposome, dexamethasone (DVD), melphalan, prednisone (MP), vindesine, carmustine, cyclophosphamide, melphalan, prednisone (M2), cyclosphosphamide, thalidomide, dexamethasone (CTD), and cisplatin, etoposide, cyclosphosphamide, prednisone (DECP). Patients with cytogenetic abnormality had lower response rate (complete response $[\mathrm{CR}]+$ near $\mathrm{CR}+$ partial response) compared with those with cytogenetic normality ( $82.1 \%$ vs 93.8\%). Patients with cytogenetic abnormality had lower $\mathrm{CR}$ rate compared with those with cytogenetic normality (32.1\% vs $50 \%$ ). The median time to CR of two groups was 3 months. The median time of sustained CR of two groups was 10 months and 12 months, respectively. Patients with cytogenetic abnormality had significant shorter survival time compared with that of cytogenetic normality (21 months vs 54 months, $P=0.027$ ) (Figure 1). Response rate and $\mathrm{CR}$ rate were $89.5 \%$ and $26.1 \%$, respectively, in patients with $1 \mathrm{q} 21$ gain. The median time of achieving $\mathrm{CR}$ and sustained $\mathrm{CR}$ was, respectively, 3 months and 7 months in patients with 1q21 gain. Median OS time was not significantly different between 1q21 amplification group and no 1q21 amplification group in patients with FISH abnormal results $(P>0.05)$ (Figure 2). Median OS time was not significantly different between $\mathrm{t}(4 ; 14)$ group and no $\mathrm{t}(4 ; 14)$ group in patients with FISH abnormal results $(P>0.05)$ (Figure 3$)$. Seven patients of $17 \mathrm{p}$ deletion died in 2 years.

\section{Discussion}

In our study, the patients with abnormal FISH results had higher frequency in stage III of ISS staging, stage III of Durie-Salmon staging, $\beta 2-\mathrm{MG}$, anemia, thrombocytopenia, hypocalcemia, elevated LDH, and elevated CRP compared with those with normal FISH results. These show that patients with cytogenetic abnormality are more serious compared with those with cytogenetic normality. CD138 expression and CD38 expression are seen in all the patients 
Table I Clinical data in patients with MM

\begin{tabular}{|c|c|}
\hline Clinical features & $\mathbf{N}$ \\
\hline \multicolumn{2}{|l|}{ Sex } \\
\hline$M / F$ & $|2 / 2|$ \\
\hline \multicolumn{2}{|l|}{ Age } \\
\hline \multicolumn{2}{|l|}{$62(38-79)$} \\
\hline \multicolumn{2}{|l|}{ D-S stage } \\
\hline I/II/IIIA/IIIB & $1 / 0 / 20 / 12$ \\
\hline \multicolumn{2}{|l|}{ ISS stage } \\
\hline I/II/III & $3 / 7 / 23$ \\
\hline \multicolumn{2}{|l|}{ lg type } \\
\hline $\operatorname{lgG} / \operatorname{lgA} /$ light chain/lgD & $9 / 10 / 11 / 3$ \\
\hline \multicolumn{2}{|l|}{ Plasmocyte } \\
\hline$\geq 30 \% /<30 \%$ & $17 / 14$ \\
\hline \multicolumn{2}{|l|}{ Immunophenotypic } \\
\hline CD38/CDI38/CD56/CD45/CD22/CD28/ & $23 / 23 / 17 / 8 / 4 / 5 / 4 / 7 / 8$ \\
\hline \multicolumn{2}{|l|}{ CDII7/CD20/CD27 } \\
\hline \multicolumn{2}{|l|}{ Bone lesion } \\
\hline$\geq 3$ sites $/<3$ sites & $32 / 1$ \\
\hline \multicolumn{2}{|l|}{$\beta 2-M G$} \\
\hline$\geq 3.5 \mathrm{mg} / \mathrm{L} /<3.5 \mathrm{mg} / \mathrm{L}$ & $22 / 8$ \\
\hline \multicolumn{2}{|l|}{ ALB } \\
\hline$<35 \mathrm{~g} / \mathrm{L} / \geq 35 \mathrm{~g} / \mathrm{L}$ & $17 / 13$ \\
\hline \multicolumn{2}{|l|}{$\mathrm{Hb}$} \\
\hline$<100 \mathrm{~g} / \mathrm{L} / \geq 100 \mathrm{~g} / \mathrm{L}$ & $17 / 13$ \\
\hline \multicolumn{2}{|l|}{ Platelet } \\
\hline$<100 \times 10^{9} / \mathrm{L} / \geq 100 \times 10^{9} / \mathrm{L}$ & $8 / 22$ \\
\hline \multicolumn{2}{|l|}{ Serum creatinine } \\
\hline$>176.8 \mu \mathrm{mol} / \mathrm{L} /<176.8 \mu \mathrm{mol} / \mathrm{L}$ & $9 / 21$ \\
\hline \multicolumn{2}{|l|}{ CRP } \\
\hline$>8 \mathrm{mg} / \mathrm{L} / \leq 8 \mathrm{mg} / \mathrm{L}$ & $10 / 16$ \\
\hline \multicolumn{2}{|l|}{ Serum calcium } \\
\hline$\geq 2.5 \mathrm{mmol} / \mathrm{L} /<2.5 \mathrm{mmol} / \mathrm{L}$ & $8 / 22$ \\
\hline Elevated LDH & $5 / 24$ \\
\hline Elevated ESR & $17 / 24$ \\
\hline Iq2I amplification & $23 / 33$ \\
\hline$t(4 p \mid 6.3 / 14 q 32)$ & $10 / 33$ \\
\hline $17 p$ deletion & $7 / 33$ \\
\hline $\mathrm{t}(14 q 32 /|| q \mid 3)$ & $5 / 33$ \\
\hline $\mathrm{t}(14 \mathrm{q} 32 / 16 \mathrm{q} 23)$ & $0 / 33$ \\
\hline
\end{tabular}

Abbreviations: M, male; F, female; D-S, Durie-Salmon; ISS, International Staging System; MG, microglobulin; ALB, albumin; Hb, hemoglobin; CRP, C-reactive protein; $\mathrm{LDH}$, lactate dehydrogenase; ESR, erythrocyte sedimentation rate; MM, multiple myeloma.

analyzed by immunotyping. The patients with abnormal FISH results have higher frequency of lacking CD45 expression compared with those with normal FISH results. Lacking CD45 expression might have been partially responsible for the worse outcomes. One study reported ${ }^{1}$ that bone marrow plasma cells strongly express CD38 and usually lack or dimly express CD45. But the mechanism action of CD38 was not clear. Strongly expressed CD138 might be associated with plasma cells homing. In our study, the patients with abnormal FISH results have higher expression of CD20, CD117, CD56, and CD28 compared with those with normal FISH results. King and Nelson ${ }^{2}$ reported that CD20

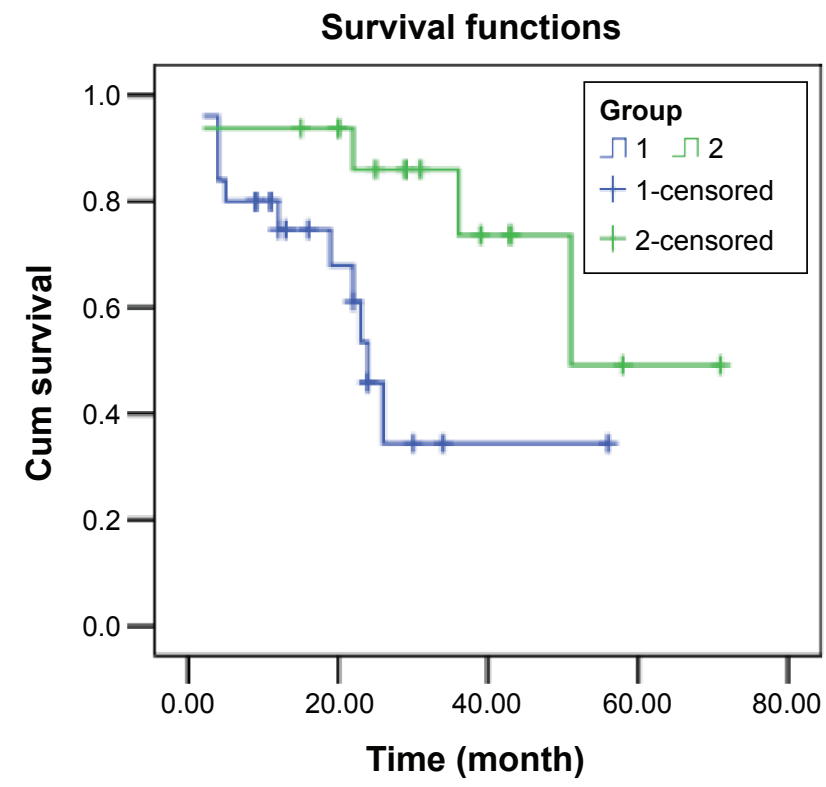

Figure I Median OS: cytogenetically abnormal (group I) and cytogenetically normal (group 2) ( $P=0.027)$.

Abbreviation: OS, overall survival.

expression was infrequent but was responsible for the poor prognosis. CD28 was responsible for the progression. CD56 was not expressed or weak in monoclonal gammopathy of undetermined significance (MGUS), but strong in MM, and was associated with poor outcomes. Shin et $\mathrm{al}^{3}$ showed that CD45-negative cases were associated with deletion 13 or aneuploidy and del(17p). CD45 negativity or CD117 negativity was associated with advanced stage.

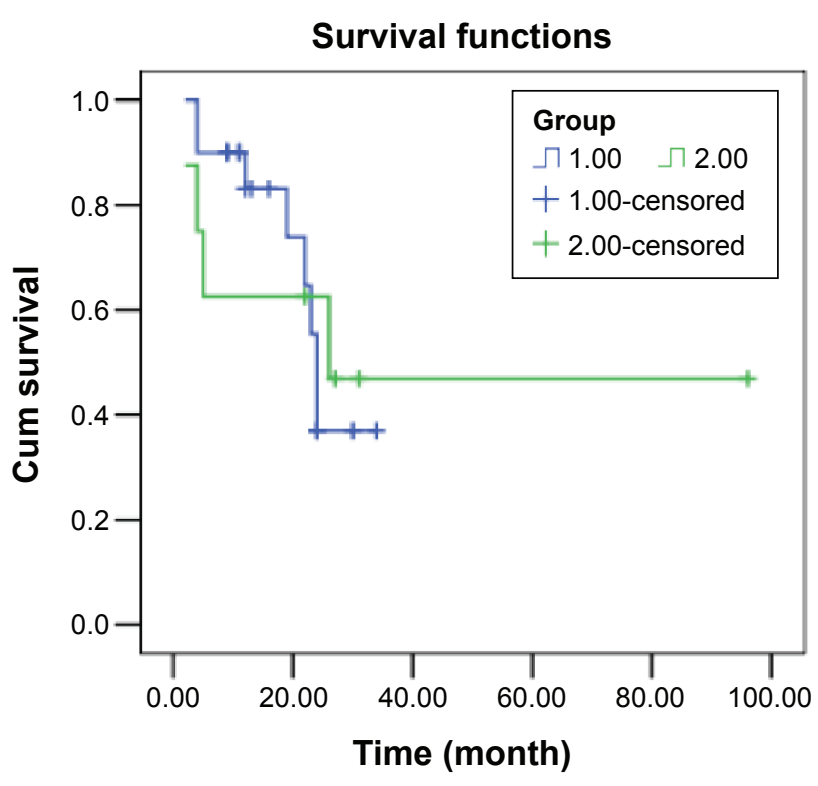

Figure 2 Median OS: Iq2I amplification (group I) and no Iq2I amplification (group 2 ) in cytogenetically abnormal patients $(P=0.917)$. Abbreviation: OS, overall survival. 


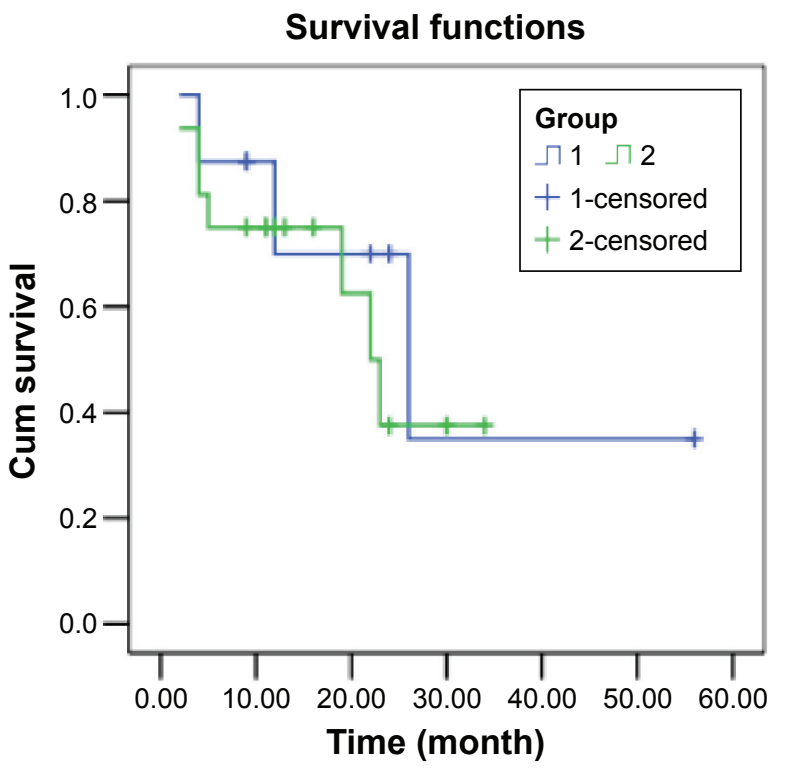

Figure 3 Median OS: $t(4 ; \mid 4)$ (group I) and no $t(4 ; \mid 4)$ (group 2 ) in cytogenetically abnormal patients $(P=0.57)$.

Abbreviation: OS, overall survival.

In our study, the patients of abnormal FISH results had higher occurrence in 1q21 amplification compared with the reports $(69.7 \%$ and $50 \%)$. Sustained CR was short in patients with 1q21 amplification, which implicated that the patients are inclined to progress. Obtaining superior proliferation in plasma cells of 1q21 was responsible for the progression. In our study, 14q32 amplification/deletion was observed in $72.7 \%$ of patients, and $t(4 ; 14)$ was the most common aberration. Lim et al ${ }^{4}$ reported that IGH rearrangements were the most common abnormality. $\mathrm{t}(4 \mathrm{p} 16.3 / 14 \mathrm{q} 32)$ rearrangements were most frequent. $t(4 ; 14)$ was an early event. The study recorded a low incidence of $17 \mathrm{p}$ deletion, which was also observed in our study. 1q21 amplification and del(17p) by FISH conferred a high incidence of progressive disease after initial therapy. Metaphase detection of IgH rearrangements and chromosome 1 aberrations concurrently was associated with a short progression-free survival. Advanced biomarker and genetic testing includes cytogenetics, FISH, and gene expression profiling to estimate the aggressiveness of the disease and personalize the patient's treatment.

It has been identified that $\mathrm{t}(4 ; 14), \mathrm{t}(14 ; 16), 13 \mathrm{q}-$, and $17 \mathrm{p}-$ are associated with poor outcomes. Our study found that the OS time of patients with abnormal FISH results was significantly shorter than those with normal FISH results. Seven patients with $17 \mathrm{q}$ deletion died in 2 years. It has been suggested that those with $17 \mathrm{p}$ deletion could rank super-high risk. We also find that three of five patients with $t(11 ; 14)$ died in 2 years, while $t(11 ; 14)$ generally is not associated with worse outcomes. Sasaki et $\mathrm{al}^{5}$ studied the impact of $\mathrm{t}(11 ; 14)$ on the outcome in patients with MM who received high-dose chemotherapy followed by auto hematopoietic cell transplantation (HCT). t $(11 ; 14)$ and HR abnormalities by conventional cytogenetics (CC) or FISH, $\beta 2-\mathrm{MG}$ of $>3.5$, and relapsed disease at the time of auto-HCT were associated with shorter OS. Patients with $\mathrm{t}(11 ; 14)$ had worse outcomes than patients with normal CC or FISH studies. But in our study, it is noteworthy that one patient with $\mathrm{t}(11 ; 14)$ harbored a 1q21 amplification, one patient harbored a $17 \mathrm{P}-$ in our study. So complex rearrangements portended a poor prognosis. $\mathrm{t}(14 ; 16)$ was found only in $7 \%$ of patients with MM by FISH. In our study, only $16 \mathrm{q} 23$ deletion/amplification was found in seven patients, and no $t(14 ; 16)$ was found. In our study, all the patients were treated by bortezomib-based chemotherapy regimens. Patients with or without $\mathrm{t}(4 ; 14)$ did not have a significant difference in median OS time. This actually suggested that bortezomib can abrogate the effects of adverse prognosticator of $t(4 ; 14)$. Inclusion of bortezomib may change the outcome of a single cytogenetic abnormality, but many patients have complex cytogenetic abnormalities. The poor outcome of patients with abnormal FISH results illustrated that bortezomib could not change the outcome of complex cytogenetic abnormalities fundamentally. Paszekova et $\mathrm{al}^{6}$ reported that $\sim 10 \%-15 \%$ of all patients with MM relapse early and have poor prognosis and outcome. Moreover, Moreau et $\mathrm{al}^{7}$ presented that the risk of early MM progression-related death was related to LDH higher than normal, International Staging System 3 (ISS3), and adverse cytogenetics $t(4 ; 14)$ and/or del $(17 p)$. Bergsagel et $\mathrm{al}^{8}$ pointed that the introduction of thalidomide, lenalidomide, and bortezomib has dramatically improved the outlook for patients with MM, but their relative benefit (or harm) for different genetic patient subgroups remains unclear. Faiman ${ }^{9}$ pointed that future goals of therapy will be to achieve minimal residual disease, biomarkers, and genomic data, which might provide a better estimate of the depth of response to therapy and OS.

\section{Disclosure}

The authors report no conflicts of interest in this work.

\section{References}

1. Witzig TE, Kimlinger TK, Ahmann GJ. Detection of myeloma cells in the peripheral blood by flow cytometry. Cytometry. 1996;26(2):113-120.

2. King MA, Nelson DS. Tumor cell heterogeneity in multiple myeloma: antigenic, morphologic, and functional studies of cells from blood and bone marrow. Blood. 1989;73(7):1925-1935.

3. Shin SY, Lee ST, Kim HJ. Antigen expression patterns of plasma cell myeloma: An Association of Cytogenetic Abnormality and International Staging System (ISS) for myeloma. J Clin Lab Anal. 2014;2(10):1002. 
4. Lim AS, Krishnan S, Lim TH. Amplification of 1q21 and other abnormalities in multiple myeloma patients from a tertiary hospital in Singapore. Indian J Hematol Blood Transfus. 2014;30(4):253-258.

5. Sasaki K, Lu G, Saliba RM. Impact of $\mathrm{t}(11 ; 14)(\mathrm{q} 13 ; \mathrm{q} 32)$ on the outcome of autologous hematopoietic cell transplantation in multiple myeloma. Biol Blood Marrow Transplant. 2013;19(8):1227-1232.

6. Paszekova H, Kryukov F, Kubiczkova L. High-risk multiple myeloma: different definitions, different outcomes? Clin Lymphoma Myeloma Leuk. 2014;14(1):24-30.

7. Moreau P, Cavo M, Sonneveld P. Combination of international scoring system 3 , high lactate dehydrogenase, and $t(4 ; 14)$ and/or del $(17 \mathrm{p})$ identifies patients with multiple myeloma (MM) treated with frontline autologous stem-cell transplantation at high risk of early MM progression-related death. J Clin Oncol. 2014;32(20):2173-2180.
8. Bergsagel PL, Mateos MV, Gutierrez NC. Improving overall survival and overcoming adverse prognosis in the treatment of cytogenetically high-risk multiple myeloma. Blood. 2013;121(6):884-892.

9. Faiman B. Myeloma genetics and genomics: practice implications and future directions. Clin Lymphoma Myeloma Leuk. 2014;14(6):436-440.

OncoTargets and Therapy

\section{Publish your work in this journal}

OncoTargets and Therapy is an international, peer-reviewed, open access journal focusing on the pathological basis of all cancers, potential targets for therapy and treatment protocols employed to improve the management of cancer patients. The journal also focuses on the impact of management programs and new therapeutic agents and protocols on

\section{Dovepress}

patient perspectives such as quality of life, adherence and satisfaction. The manuscript management system is completely online and includes a very quick and fair peer-review system, which is all easy to use. Visit http://www.dovepress.com/testimonials.php to read real quotes from published authors.

Submit your manuscript here: http://www.dovepress.com/oncotargets-and-therapy-journal 